\title{
Quantifying the Polymeric Capping of Nanoparticles with X-Ray Photoelectron Spectroscopy
}

Eden E. L. Tanner, ${ }^{1 \dagger}$ Stanislav V. Sokolov, ${ }^{1}$ Kamonwad Ngamchuea, ${ }^{1}$ Robert G. Palgrave, ${ }^{2}$ Richard G. Compton ${ }^{1 *}$

1 University of Oxford, Department of Chemistry, Physical and Theoretical Chemistry Laboratory, Oxford University, South Parks Road, Oxford, OX1 3QZ, United Kingdom.

2 Department of Chemistry, University College London, London, UK

$\dagger$ Now at the School of Engineering \& Applied Sciences, Harvard University, Cambridge, MA, USA

* Corresponding author: Richard G. Compton, Department of Chemistry, Physical \& Theoretical Chemistry Laboratory, University of Oxford, South Parks Road, Oxford, OX1 3QZ, United Kingdom

Emails: richard.compton@chem.ox.ac.uk

Tel: +44(0)1865275957Ｆax: +44(0)1865275410

\begin{abstract}
X-ray Photoelectron Spectroscopy was used to characterise silver nanoparticles capped with poly(ethylene) glycol in a room temperature ionic liquid (RTIL), 1-butyl-3methylimidazolium tetrafluoroborate $\left([\mathrm{Bmim}]\left[\mathrm{BF}_{4}\right]\right)$. The amounts of oxygen and silver present in nanoparticles capped with different molecular weight thiolated PEG chains were monitored, and the number of thiolated PEG chains per nanoparticle was calculated, an in situ characterisation not previously possible.
\end{abstract}




\section{Main Text}

The capping of nanoparticles controls a large number of their properties, including reactivity, ${ }^{[1]}$ stability, ${ }^{[2]}$ and susceptibility to electron transfer. ${ }^{[3]}$ However, there is a lack of detailed understanding of the structure and the extent to which a nanoparticle is capped, particularly in situ in solution. The characterisation of the capping agents in situ is highly important for the development of the controlled synthesis of nanoparticles and the use of the nanoparticles in a broad range of fields including nanocatalysis ${ }^{[4]}$ and biomedical applications. ${ }^{[5]}$

X-ray Photoelectron Spectroscopy (XPS) is a powerful surface characterisation technique frequently applied to quantitatively understand solid materials. However, recently XPS has been used to analyse samples of ionic liquids (ILs), ${ }^{[6]}$ which have such low volatilities that they can be used in the high vacuum environment of the instrument, allowing characterisation

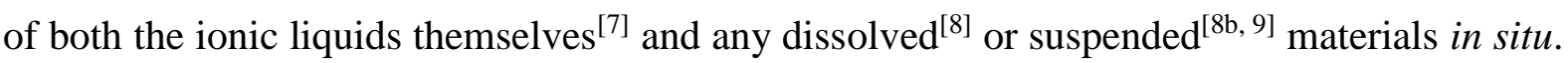
As ILs have a number of desirable physicochemical properties such as recyclability, tuneable hydrophilicity and miscibility as well as wide electrochemical windows, ${ }^{[10]}$ ILs have been used as an alternative medium for many processes. In particular, ILs have been demonstrated to enhance the physical and chemical properties of nanoparticles and stabilize the nanoparticle catalysts. ${ }^{[1]}$ The use of ionic liquid in XPS measurement also facilitates studies of metal deposition in situ, for example potassium ${ }^{[12]}$ and rubidium ${ }^{[13]}$ deposition, allowing the investigation of the fundamental kinetic and thermodynamic properties of the processes.

This work seeks to use XPS to quantify the polymeric capping of silver nanoparticles suspended in a Room Temperature Ionic Liquid, 1-butyl-3-methylimidazolium tetrafluoroborate $\left([\mathrm{Bmim}]\left[\mathrm{BF}_{4}\right]\right)$. Thiolated poly(ethlyene) glycol (PEG) is used as a proofof-concept example to demonstrate the developed technique. PEG has been frequently used as a capping agent due to its acid resistance and customisability. ${ }^{[14]}$

The amounts of silver and oxygen atoms present are monitored, allowing the first insight into the number of thiolated PEG chains attached to Ag NPs in solution. The wrapping of thiolated PEG around the Ag NP is potentially crucial in protecting the silver core against dissolution and other chemical reactions. In an electrochemical context, the capping molecules are thought to reversibly partially 'unwrap' to allow electrical contact to be made between the nanoparticle core and the electrode. ${ }^{[1]}$ 
$[\mathrm{Bmim}]\left[\mathrm{BF}_{4}\right]$

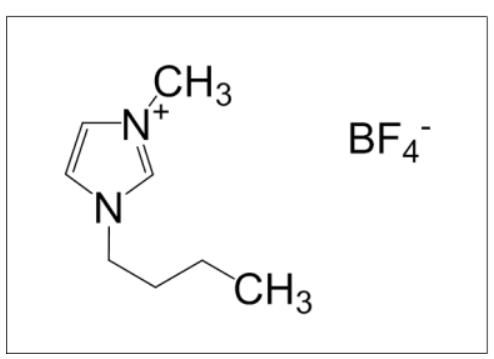

Thiolated PEG

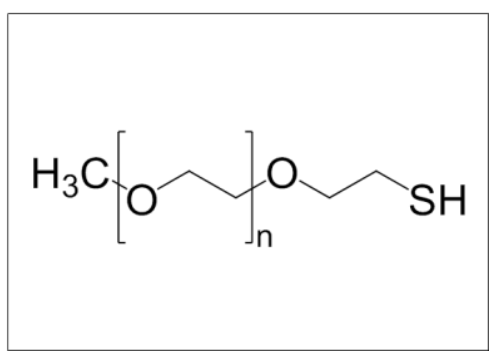

Figure 1: Chemical structures of $[\mathrm{Bmim}]\left[\mathrm{BF}_{4}\right]$ and thiolated PEG.

First, the XPS spectra of pure ionic liquid in the absence of nanoparticles were recorded. The survey of the pure ionic liquid across the binding energies shown in Figure 2 shows that all the expected peaks characteristic of the components of the ionic liquid (carbon, boron, nitrogen, and fluorine) are observed. The slight variation in binding energy $( \pm 0.1 \mathrm{eV})$ between samples was attributed to charging. Notably, in the blank IL only an extremely weak O1s peak is observed at a binding energy of around $530 \mathrm{eV}$, quantified as 0.6 atomic\% oxygen, indicating that the IL has a low surface water content. The atomic\% composition of the blank IL is shown in Table 1. The measured values are close to the expected for all elements, and comparable with analyses of this IL performed by others. ${ }^{[7 c, 15]}$ We estimate the relative error in compositions as $+/-10 \% .{ }^{[6]}$ The sample is found to be slightly richer in $\mathrm{F}$ and $\mathrm{B}$ and slightly deficient in $\mathrm{C}$ and $\mathrm{N}$ compared with the expected values from the molecular structure. Since B and F are both found in the anion and C and N both found in the cation, this may indicate that the surface of the IL has a slight preference for the anionic species. 


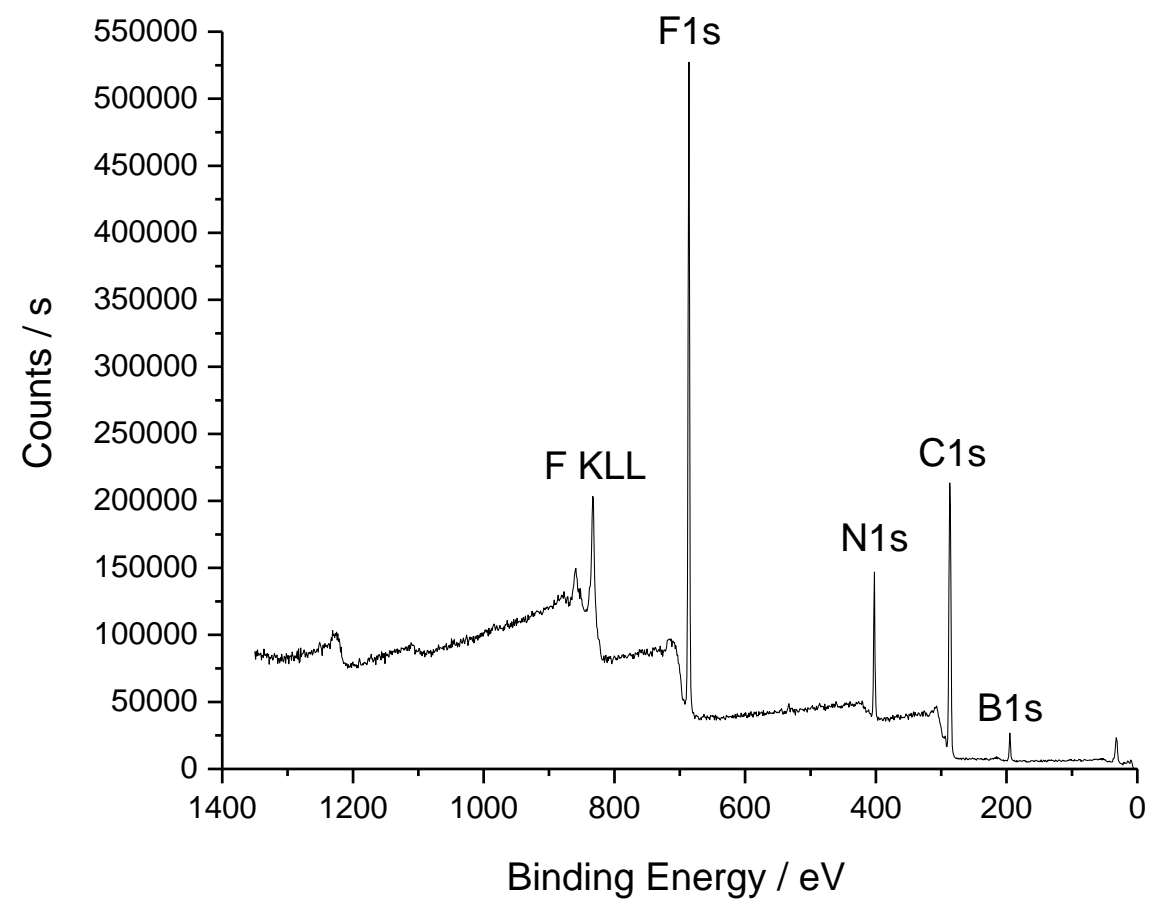

Figure 2: A survey XPS scan of pure [Bmim][BF4].

Table 1. Measured compositions of IL samples by XPS. Error is XPS composition estimated as $+/-10 \%$ of each value.

\begin{tabular}{|l|l|l|l|l|l|}
\hline & \multicolumn{5}{|c|}{ Atomic\% } \\
\hline Element & $\begin{array}{l}\text { Molecular } \\
\text { structure of IL }\end{array}$ & Blank IL & $\begin{array}{l}\text { IL + PEG } \\
\mathbf{2 0 0 0} \text { MW }\end{array}$ & $\begin{array}{l}\text { IL + Ag + } \\
\text { PEG 2000 } \\
\text { MW }\end{array}$ & $\begin{array}{l}\text { IL Ag + } \\
\text { PEG 6000 } \\
\text { MW }\end{array}$ \\
\hline $\mathrm{B}$ & 6.7 & 7.1 & 8.1 & 6.9 & 6.4 \\
\hline $\mathrm{C}$ & 53.3 & 49.2 & 51.8 & 48.1 & 48.9 \\
\hline $\mathrm{N}$ & 13.3 & 11.8 & 12.1 & 11.6 & 11.9 \\
\hline $\mathrm{O}$ & 0 & 0.6 & 0.6 & 1.1 & 1.2 \\
\hline $\mathrm{F}$ & 26.7 & 31.2 & 27.4 & 31.8 & 31.4 \\
\hline $\mathrm{Ag}$ & 0 & 0 & 0 & 0.5 & 0.2 \\
\hline
\end{tabular}

A sample of IL containing poly(ethylene) glycol methyl ether thiol (thiolated-PEG) with molecular weight 2000 Dalton was prepared by combining $1.8 \mu \mathrm{L}$ of a $2 \mathrm{mg} / \mathrm{mL}$ solution of thiolated PEG into $30 \mu \mathrm{L}$ of $[\mathrm{Bmim}]\left[\mathrm{BF}_{4}\right]$ (99\%, IoLiTec, Germany) and placing it under 0.1 mbar vacuum overnight to remove any water present. XPS of this sample gave spectra very similar to the blank IL. As can be seen in Table 1, the IL containing PEG 2000 MW showed a slightly higher carbon surface content (51.8 atomic\% vs. 49.2 atomic\% in the blank 
IL). However the oxygen level was identical, and if PEG were present at the surface, the oxygen level would be expected to increase. Therefore it seems that addition of PEG 2000 MW to the IL does not result in detectable PEG at the surface of the droplet by XPS. Instead we ascribe the increased $\mathrm{C} 1 \mathrm{~s}$ signal to environmental contamination which is a common feature for samples analysed by XPS.

Next, the XPS responses of ionic liquid in the presence of Ag NPs were studied. Citrate capped nanoparticles were prepared as described previously, ${ }^{[16]}$ and recapped with poly(ethylene) glycol methyl ether thiol (thiolated PEG, $2000 \mathrm{MW}$ or $6000 \mathrm{MW}$ ) by placing $10 \mu \mathrm{L}$ of a $2 \mathrm{mg} / \mathrm{mL}$ solution of thiolated PEG into a $490 \mu \mathrm{L}$ solution of $20 \mathrm{~nm}$ (diameter) nanoparticles, and allowed to sit for three hours. Then, an aliquot of nanoparticles $(90 \mu \mathrm{L})$ was combined with $30 \mu \mathrm{L}$ of $\left[\mathrm{Bmim}_{[}\right]\left[\mathrm{BF}_{4}\right]$ (99\%, IoLiTec, Germany) and placed under 0.1 mbar vacuum overnight to remove the water and other volatile impurities, resulting in a solution of $2 \times 10^{-5} \mathrm{~mol} \mathrm{~m}^{-3} \mathrm{Ag}$ NPs. All aqueous solutions and nanoparticle suspensions were made using deionized water from Millipore with resistivity not less than $18.2 \mathrm{M} \Omega \mathrm{cm}$ at $25{ }^{\circ} \mathrm{C}$.

A $5 \mu \mathrm{L}$ droplet of the NP-containing IL was placed on a borosilicate glass slide and analysed by XPS; detailed in SI. The droplets measured around $3 \mathrm{~mm}$ in diameter. The resulting XP spectra of the sample showed indistinguishable $\mathrm{C} 1 \mathrm{~s}, \mathrm{~N} 1 \mathrm{~s}, \mathrm{~F} 1 \mathrm{~s}$ and $\mathrm{B} 1 \mathrm{~s}$ peaks from the blank IL. Samples containing Ag NPs capped with thiolated PEG (2000 MW or $6000 \mathrm{MW}$ ) in $[\mathrm{Bmim}]\left[\mathrm{BF}_{4}\right]$ ionic liquid show additional peaks as compared with the pure ionic liquid or the IL containing PEG 2000 MW. Figure 3 shows the O1s peaks in the blank IL and IL containing Ag NPs capped with 2000 and 6000 MW thiolated PEG. The O1s signal clearly increases in intensity on addition of the Ag NPs. This is reflected in the increase in $\mathrm{O}$ concentration for these samples shown in Table 1. Figure 4 shows the $\mathrm{Ag} 3 \mathrm{~d}$ peaks recorded from the Ag NP containing samples. Ag can clearly be detected. The Ag $3 \mathrm{~d}$ peaks are observed at binding energies of 375 and $369 \mathrm{eV}$ corresponding to $\mathrm{Ag} 3 \mathrm{~d}_{3 / 2}$ and $\mathrm{Ag} 3 \mathrm{~d}_{5 / 2}$ peaks from metallic silver. As expected, silver is only detectable by XPS in samples containing Ag NPs. In the case of the $2000 \mathrm{MW}$ PEG, the observed O:Ag ratio was $1.8 \pm 0.2$, whilst in the case of the $6000 \mathrm{MW}$ PEG, the observed surface O:Ag ratio in the surface layer was $4.3 \pm$ 0.4 . 


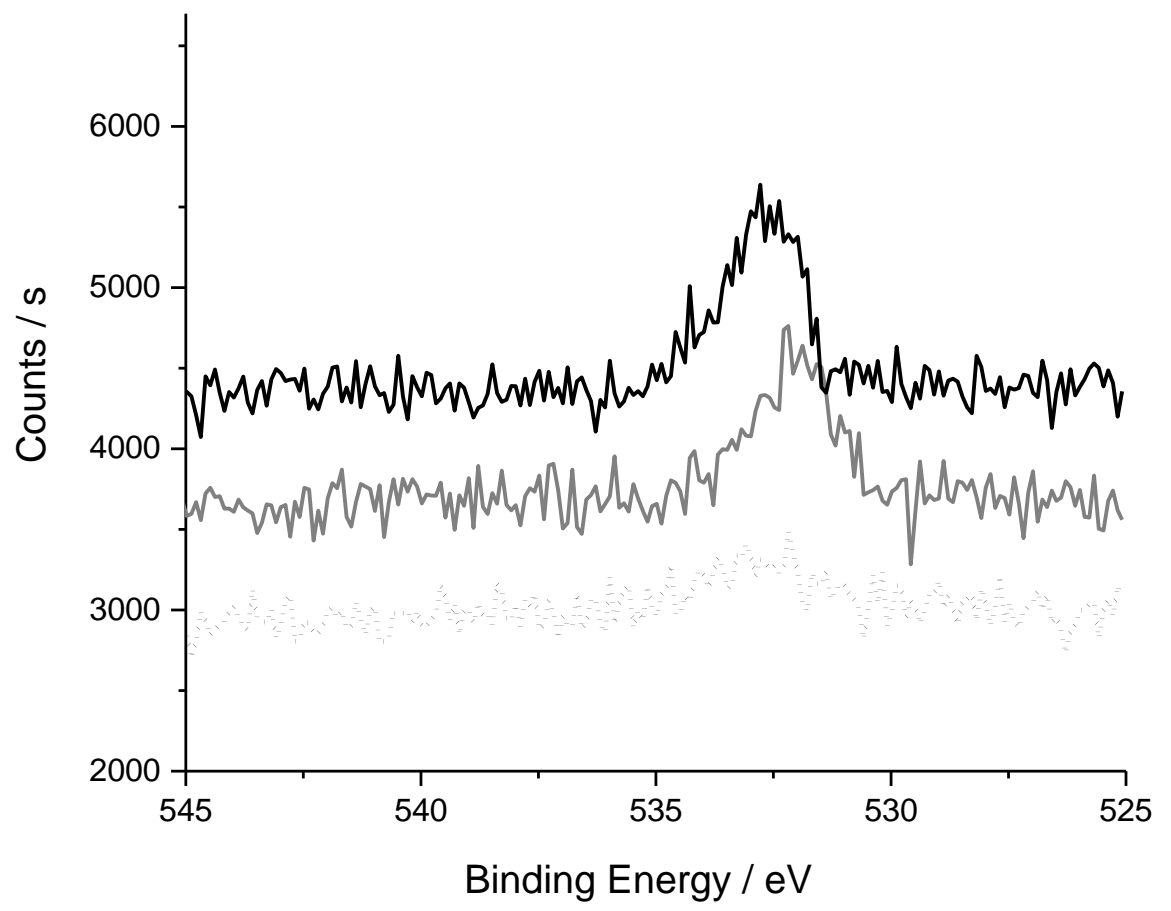

Figure 3: Oxygen 1s signals of blank ionic liquid (dotted line), $2000 \mathrm{MW}$ thiolated PEG capped Ag NPs (grey solid line) and 6000 MW thiolated PEG capped Ag NPs (black solid line) as detected by XPS.

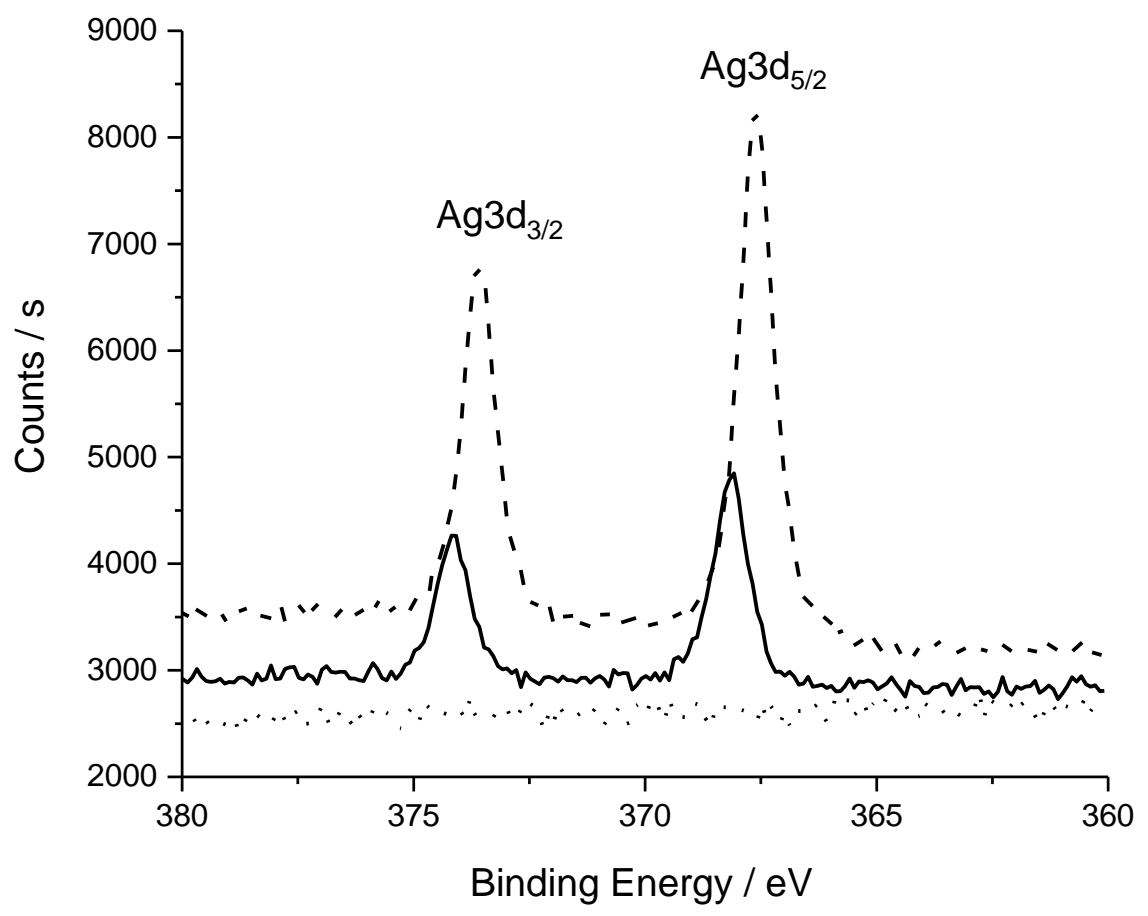

Figure 4: Ag 3d signals of blank ionic liquid (dotted line), 2000 MW thiolated PEG capped Ag NPs (dashed line) and 6000 MW thiolated PEG capped Ag NPs (black solid line) as detected by XPS. 
The difference in the ratio of silver to oxygen present reflects the length of the thiolated PEG chain, with each 6000 MW thiolated PEG chain containing 135 oxygen atoms, and each 2000 MW thiolated PEG chain containing only 44 oxygen atoms. We can therefore use the XPS measurement to determine how many thiolated PEG chains are attached to each nanoparticle. This is done by calculating the number of silver atoms in each nanoparticle, multiplying this number by the $\mathrm{Ag}: \mathrm{O}$ ratio in each case to obtain the number of oxygen atoms present, and dividing by the number of oxygen atoms in each thiolated PEG chain of the given molecular weights. From this, $1.0 \pm 0.1 \times 10^{4}$ thiolated PEG chains of $2000 \mathrm{MW}$ and $7.7 \pm 0.8 \times 10^{3}$ thiolated PEG chains of $6000 \mathrm{MW}$ are attached to the silver nanoparticles. This determination is an upper limit, as it is possible that some free PEG is present in the samples, although this was not detected in the PEG containing IL in the absence of Ag NPs. A simple estimation of the number of chains that make up a saturated monolayer - calculated by dividing the surface area of a nanoparticle by the van der Waals radius of a sulfur atom - is $1.2 \times 10^{5}$ chains. This suggests that the thiolated PEG chains are not very tightly packed around the silver nanoparticle.

In conclusion, XPS has been used to characterise thiolated PEG capped Ag NPs in a RTIL by quantifying the ratio of silver atoms to oxygen atoms. This analysis allowed the calculation of the number of thiolated PEG chains per nanoparticle as $1.0 \pm 0.1 \times 10^{4}$ thiolated PEG chains of $2000 \mathrm{MW}$ or $7.7 \pm 0.8 \times 10^{3}$ thiolated PEG chains of $6000 \mathrm{MW}$.

\section{Supporting Information}

The Supporting Information includes the detail of chemical reagents, instrumentation, measurement procedures and the characterisation of Ag NPs.

\section{Acknowledgements}

The research leading to these results has received partial funding from the European Research Council under the European Union's Seventh Framework Programme (FP/2007-2013)/ERC Grant Agreement no. [320403]. The XPS facility at UCL is supported by EPSRC grant $\mathrm{EP} / \mathrm{P} 030084 / 1$. 


\section{References}

[1] E. E. L. Tanner, K. Tschulik, R. Tahany, K. Jurkschat, C. Batchelor-McAuley, R. G. Compton, J. Phys. Chem. C 2015, 119, 18808-18815.

[2] M. Tejamaya, I. Romer, R. C. Merrifield, J. R. Lead, Environ. Sci. Technol. 2012, 46, 70117017.

[3] E. E. L. Tanner, S. V. Sokolov, N. P. Young, R. G. Compton, Phys. Chem. Chem. Phys. 2017, 19, 9733-9738.

[4] Z. Niu, Y. Li, Chem. Mater. 2013, 26, 72-83.

[5] K. Niska, N. Knap, A. Kedzia, M. Jaskiewicz, W. Kamysz, I. Inkielewicz-Stepniak, Int. J. Med. Sci. 2016, 13, 772-782.

[6] K. R. Lovelock, I. J. Villar-Garcia, F. Maier, H. P. Steinruck, P. Licence, Chem. Rev. 2010, $110,5158-5190$.

[7] a) N. E. Cousens, L. J. Taylor Kearney, M. T. Clough, K. R. Lovelock, R. G. Palgrave, S. Perkin, Dalton Trans. 2014, 43, 10910-10919; b) L. S. Longo, E. F. Smith, P. Licence, ACS Sustainable Chem. Eng. 2016, 4, 5953-5962; c) E. F. Smith, F. J. Rutten, I. J. Villar-Garcia, D. Briggs, P. Licence, Langmuir 2006, 22, 9386-9392.

[8] a) D. S. Silvester, T. L. Broder, L. Aldous, C. Hardacre, A. Crossley, R. G. Compton, Analyst 2007, 132, 196-198; b) I. Niedermaier, C. Kolbeck, N. Taccardi, P. S. Schulz, J. Li, T. Drewello, P. Wasserscheid, H. P. Steinruck, F. Maier, ChemPhysChem 2012, 13, 1725-1735.

[9] a) P. Licence, Angew. Chem., Int. Ed. Engl. 2012, 51, 4789-4791; b) F. Bernardi, J. D. Scholten, G. H. Fecher, J. Dupont, J. Morais, Chem. Phys. Lett. 2009, 479, 113-116.

[10] a) A. M. O'Mahony, D. S. Silvester, L. Aldous, C. Hardacre, R. G. Compton, J. Chem. Eng. Data 2008, 53, 2884-2891; b) P. Bonhôte, A.-P. Dias, N. Papageorgiou, K. Kalyanasundaram, M. Grätzel, Inorg. Chem. 1996, 35, 1168-1178.

[11] Z. He, P. Alexandridis, Adv. Colloid Interface Sci. 2017, 244, 54-70.

[12] R. Wibowo, L. Aldous, R. M. J. Jacobs, N. S. A. Manan, R. G. Compton, Chem. Phys. Lett. 2011, 509, 72-76.

[13] R. Wibowo, L. Aldous, R. M. J. Jacobs, N. S. A. Manan, R. G. Compton, Chem. Phys. Lett. 2011, 517, 103-107.

[14] a) P. Kingshott, H. Thissen, H. J. Griesser, Biomaterials 2002, 23, 2043-2056; b) V. C. F. Mosqueira, P. Legrand, J. L. Morgat, M. Vert, E. Mysiakine, R. Gref, J. P. Devissaguet, G. Barratt, Pharm. Res. 2001, 18, 1411-1419; c) T. Maldiney, C. Richard, J. Seguin, N. Wattier, M. Bessodes, D. Scherman, ACS nano 2011, 5, 854-862; d) D. Pozzi, V. Colapicchioni, G. Caracciolo, S. Piovesana, A. L. Capriotti, S. Palchetti, S. De Grossi, A. Riccioli, H. Amenitsch, A. Lagana, Nanoscale 2014, 6, 2782-2792.

[15] V. Lockett, R. Sedev, C. Bassell, J. Ralston, Phys. Chem. Chem. Phys. 2008, 10, 1330-1335.

[16] E. J. E. Stuart, K. Tschulik, J. Ellison, R. G. Compton, Electroanalysis 2014, 26, 285-291. 
